\section{A lupus explanation}

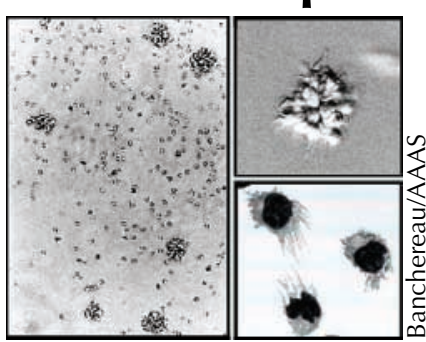

Serum from a lupus patient turns monocytes into dendritic cells. tissues.
The cellular defect that underlies the autoimmune disease lupus may finally have been uncovered. Overactive dendritic cells may be inciting attacks on the patient's own

In lupus, the immune system batters the skin, kidneys, brain, and other organs, but the stimulus for the attack has eluded explanation. Jacques Banchereau (Baylor Institute for Immunology Research, Dallas, TX) and colleagues found that serum from lupus patients spurs monocytes to mature into dendritic cells that may trigger an autoimmune reaction. The dendritic cells gobbled up fragments of dead body cells and presented them to autologous CD4 T cells.

What stimulates the maturation of monocytes? The research implicated $\alpha$ interferon, which is produced by one class of dendritic cells and is elevated in people with lupus. According to Banchereau, the results suggest that hyperactive dendritic cells, driven by high levels of $\alpha$ interferon, may cause lupus.

That still leaves a puzzle, however. How to explain the high levels of interferon, especially when the interferon-producing dendritic cells are often scarce in the blood of lupus patients? Banchereau thinks that armies of dendritic cells have deployed from the blood into the tissues. There, if they are simulated by a virus, the errant cells may start pumping out $\alpha$ interferon, leading to the self-destructive attacks that produce symptoms like vasculitis and rashes.

Patients could soon benefit from this work, Banchereau predicts. Steroids and immunosuppressants can ease symptoms of lupus, but they cannot cure the disease. "This provides us with a target for therapeutic intervention," he says.

Reference: Blanco, P., et al. 2001. Science. 294:1540-1543.

\title{
Kiss of death
}

D8 cytotoxic $\mathrm{T}$ cells are serial killers that slay one infected or cancerous cell after another. During each fatal encounter, an intercellular junction called the immunological synapse forms between killer and victim, according to researchers from Oxford University (Oxford, UK). Their work suggests new functions for the immunological synapse and supports the hypothesis that the immune system governs the number of cytotoxic T cells by "retiring" veterans of multiple battles.

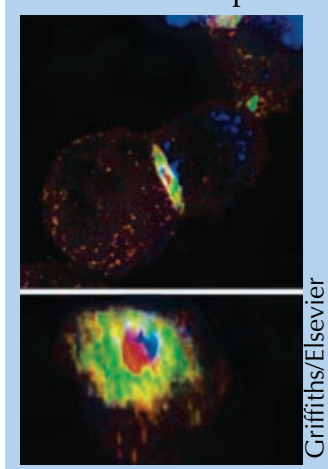

Lytic granules (blue) insert between the signaling patch (red) and the adhesion ring (green).
In earlier studies immunological synapses were spotted only when an antigenpresenting cell met and activated a CD4 T cell. Although a CD8 cytotoxic T cell may attach to its victim for as little as two minutes, Gillian Griffiths and colleagues observed that synapses also formed during these contacts. The synapses have the characteristic bull's eye structure of a ring of adhesion proteins surrounding a ring of signaling proteins. The authors also showed that the cell-killing granules released by the cytotoxic $\mathrm{T}$ lymphocyte emerged from a focused secretory domain within the adhesion domain.

The immunological synapse was thought to provide sustained signaling. But the new results suggest that the architecture of the synapse may help guide the secretion of molecules and prevent the death of nearby bystander cells, Griffiths says. She adds that the synapse may also participate in regulating the numbers of cytotoxic T cells. As the two cells part, the T cell picks up some of the victim's proteins, probably by ripping off a hunk of membrane. Thus, the $\mathrm{T}$ cells end up looking more and more like their targets, leading them to destroy each other in a phenomenon called fratricide. References: Stinchcombe, J.C., et al. 2001. Immunity. 15:751-761.

\section{Growing together}

$\mathrm{T}^{\mathrm{a}}$ alk about codependence. In the developing Drosophila nerve cord, neurons and glia cannot survive without each other. Kill a neuron, and its supporting glia will die, and vice versa. New research illuminates one of the molecular mechanisms behind this relationship. Neurons produce a protein that keeps glial cells alive, and in the process help shape the growing nervous system.

Alicia Hidalgo (Cambridge University, Cambridge, UK) and colleagues determined the effects of the protein Vein on survival of glial cells in the developing ventral nerve cord of Drosophila. The protein is made in the pioneer neurons that lay down the scaffolding for the nerve cord.
Blocking Vein production or blocking its receptors kills the glia near the pioneer neurons, the authors found. They also showed that Vein acts by activating the Ras/MAP-kinase pathway in those glia.

Whereas most neurons require glia for survival, pioneer neurons depend on glia to guide their growth, not for survival. As the nervous system develops, glia help to establish the trajectories of the pioneers' axons by stationing themselves at key points where the axons "decide" which direction to steer or to which partner axon to adhere, Hidalgo says. Normal flies produce far too many glia, many of which eventually die. Which cells survive helps determine the pattern of axon trajectories, and Vein may thus indirectly mold neural pathways.

"One of the implications of this work is that the insect nervous system is just as plastic as the vertebrate nervous system," Hidalgo says. She ventures

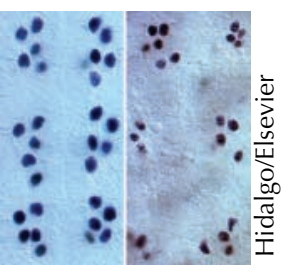

Less production of Vein by neurons (right) leads to glial death. that Drosophila might even be a good model for studying processes once thought to need a vertebrate model, such as regrowth of a damaged spinal cord.

Reference: Hidalgo, A., et al. 2001. Dev. Cell. 1:679-690. 